\title{
Best Position Method for Experimental Data Acquisition Systems Using Low-cost GPS Receiver
}

\author{
I. Abba ${ }^{1}$, W.A.W.Z. Abidin ${ }^{1}$, A. K. Othman ${ }^{1}$, K. H. Ping ${ }^{1}$, M. Abdullah ${ }^{2}$ and B. Voon Pai ${ }^{1}$ \\ ${ }^{1}$ Department of Electronic Engineering, Universiti Malaysia Sarawak, \\ 94300 Kota Samarahan, Sarawak, Malaysia \\ ${ }^{2}$ Department of Electrical, Electronic \& Systems Engineering/Institute of Space Science, Universiti Kebangsaan Malaysia, \\ 43600 UKM Bangi, Selangor, Malaysia \\ ibrahimaba12950@yahoo.com
}

\begin{abstract}
Mobile satellites (MS) propagation is important for the study of satellites signal strength. This paper concentrates on the evaluation of best positioning for the experimental data acquisition system, where a portable commercial Global Positioning System receiver was used in getting the best position. The receiver azimuth angle and elevation angle were utilized in obtaining the best results. The experiment is carried out in an open space area where line-of sight will be visible. Measurement is then performed to determine the relationship between the signal performance with respect to the elevation and azimuth angles. The result obtained from this experiment will be used to set a standard and best positioning for the GPS receiver in collecting data for experimental purpose. Single frequency receivers have the advantage that they are low-cost and CA-code acquisition for civilian access provide higher signal to noise ratio than synthesized P-code acquisition. Since the civilian users can have only access of CA-code, while the P-code in mainly for military users, this kind of receiver was used in this research. Details methodology for doing the experimental works will be discussed. The experiment was conducted at roof top of faculty of engineering building, Universiti Malaysia Sarawak. Therefore, a handheld GPS receiver best positioning experiment designed in order to encourage more experimental works to be carried out in the less developed countries. The analysis of the signal performance under different MS environments is performed with respect to the information such as elevation and azimuth angles.
\end{abstract}

Keywords-component; Handheld GPS receiver; data acquisition systems; mobile satellite

\section{INTRODUCTION}

Experimental data for mobile satellite (MS) signal important in communication services. MS system was designed to promote communication to areas where terrestrial communications is not available. This serve as an alternative to the current communication system which normally has a potential to be develop again to support the ever increasing demand of personal and multimedia communication. The portable Global Positioning System (GPS) receivers are increasingly popular among the outdoor user, though the handheld GPS receiver prices have dropped so anybody can afford to buy it.

Communication satellites function as a microwave repeater station for the exchanging of information between the users in different forms [1]. However, GPS is best known as a worldwide positioning system and the main purpose is to provide accurate positioning location at all points on the earth's surface at all times [2,3]. It is intended mainly for defense purposes but the civilian community now constitutes the bulk of users. The satellites constellation comprises 24 satellites such that at least 4 satellites are visible everywhere on earth at any time. The orbits are essentially circular at an altitude of about $20,200 \mathrm{~km}$, with orbital inclinations of $63^{\circ}$ and with $12 \mathrm{~h}$ (sidereal time) duration [4]. The nominal orbital period of GPS satellite is 11 hours, 58 minutes, 4.1 seconds $[4,5]$. This means that each GPS satellite orbits the earth twice each day.

The GPS signals consist of two carrier frequencies L1:1575.42 MHz (0.19029 m wavelength) C/A-Code (Code acquisition) and L2: $1227.60 \mathrm{MHz}(0.24421 \mathrm{~m}$ wavelength) which normally controlled by the military users with basic signal of higher precision $[6,7]$.

The commercial handheld GPS receiver used in the experiment was Garmin receiver. The receiver antenna radiation patterns indicates that the antenna gain is $5 \mathrm{dBi}$ [8] for a typical micro strip patch antenna having right-hand circularly polarized (RHCP). In order to have high-position accuracy experimental result, a positional experiment was conducted using open space position method. Many researches were conducted on held GPS receiver for precise positioning method mostly the research were on geo-catching, site location and point positioning [9]. This research was based on the data acquisition for experimental method. The receiver used for this experiment was not meant for laboratory purposes but it was designed mainly for navigational and position location [10]. The precise position technique in this paper employed two different approaches, the receiver azimuth position and elevation angle position to get the best experimental technical data.

The signal performance of the MS is affected by many factors such as ionosphere effect, trees shadowed, building shadowed and multipath effect, but this experimental research will focus on the satellite signals for an open space environment in which the receiving satellite signal does not experience significant fading effect due to building or trees.

Handheld receivers are used for positioning and geocatching using DGPS-service or WASS/EGNOS signals. This position is realized using code pseudo-range $[9,11]$. By using Garmin handheld receiver the phase and code information 